\title{
EFECTIVIDAD DE LA APLICACIÓN DE BIOINSUMO DE AGUAS RESIDUALES DE CAFÉ EN PRODUCTIVIDAD DE HORTALIZAS
}

\section{EFFECTIVENESS OF THE IMPLEMENTATION OF COFFEE WASTEWATERBIOSUPPLIESINPRODUCTIVITYOFVEGETABLES}

\author{
Eyvar Andrés Bolaños ${ }^{1}$, Martha C. Muelas², Lorena M. Mejia², Tatiana Trochez² \\ Recibido para publicación: Noviembre 15 de 2012 - Aceptado para publicación: Marzo 192013
}

\begin{abstract}
RESUMEN
El café de Colombia se caracteriza por su calidad organoléptica, pero su proceso de tratamiento de beneficio húmedo se constituye en una problemática debido a la cantidad de agua utilizada y la contaminación que generan los subproductos como residuos sólidos y aguas residuales, generando desequilibrios ecológicos y problemas de salud. Por lo anterior, este proyecto pretendió probar la efectividad de las aguas residuales de café tratadas con lixiviado proveniente de pulpa de café inoculado con microorganismos eficientes (ME) como bioinsumo, para la producción de rábano, Raphanus sativus y pepino, Cucumis sativus. Se realizaron ensayos con cinco tratamientos y tres repeticiones conducentes a evaluar la dosis volumétrica adecuada del bioinsumo que presente los mejores índices de productividad en rábano. Una vez determinada la dosis ideal se procedió a la comparación de la misma con un fertilizante de síntesis química mediante un ensayo con tres tratamientos y tres repeticiones en pepino. Los resultados demuestran que la dosis más adecuada de aguas residuales tratadas con ME para utilizarse como bioinsumo en rábano y pepino fue $30 \mathrm{~cm}^{3} \mathrm{~L}^{-1}$ de agua; Igualmente hubo diferencias significativas en la producción de pepino cuando se compara el bioinsumo con el testigo. Se espera que el uso de microorganismos eficientes sea una alternativa ambientalmente sostenible, para la descontaminación de aguas y el mejoramiento de la productividad agrícola.
\end{abstract}

Palabras clave: microorganismos eficientes, bioinsumo, Coffea arabica, aguas residuales.

\begin{abstract}
Colombia's coffee is characterized by its organoleptic quality, but the humid beneficiary process constitutes a problem because of the amount of water used and the pollution generated by products as solid waste and wastewater, creating ecological imbalances and health problems. This project was intended to test the effectiveness of the wastewater treated with coffee leachate from coffee pulp inoculated with efficient microorganisms (EM), used as biosupplie for the production of radish Raphanus sativus and cucumber Cucumis

\footnotetext{
${ }^{1}$ Magister en ciencias con énfasis en Recursos Fitogenéticos, Director de programa, docente de fisiología vegetal y Botánica y sistemas de producción Agrícola - Institución Universitaria Tecnológica de Comfacauca - Unicomfacauca. Santander de Quilichao, Cauca. Colombia Cra 9 -5 - 53, Telefax: 8298729. abolanos@unicomfacauca.edu.co

2Estudiantes Tecnología Agroambiental, Unicomfacauca
} 
sativus. Some tests were carried out to evaluate the appropriate biosupplie volumetric dosage that presents the best productivity rates in radish. Five treatments with three repetitions were made. Once the ideal dosage was determined, it was compared with a synthetic chemical fertilizer in an experiment of three treatments and three repetitions in cucumber. The results show that the most suitable dosage of wastewater treated with ME as biosupplie to be used in radish and cucumber was $30 \mathrm{~cm}^{3} \mathrm{~L}^{-1}$; likewise, significant differences in cucumber production when compared the biosupplie with control group were found. It is expected that the use of efficient microorganisms could be an environmentally sustainable alternative for decontamination of water and improving the agricultural productivity.

Key words: efficient microorganisms, biosupplie, Coffea arabica, wastewater.

\section{INTRODUCCIÓN}

de contaminación de la industria cafetera y

La caficultura es una de las principales actividades agrícolas de Colombia y su grano es considerado de alta calidad en todo el mundo por sus características organolépticas (FEDECAFE 2009). Por esta razón la Federación Nacional de Cafeteros ha estimulado la siembra y renovación de cafetales debido a que es un producto con mercado asegurado y de buena rentabilidad.

En el país el grano de café se procesa por vía húmeda, el fruto después de ser cosechado se envía al beneficio donde se separa la pulpa del grano con máquinas despulpadoras y posteriormente se fermenta de 12 a 18 horas horas; este tipo de tratamiento asegura una alta calidad organoléptica, sin embargo tiene sus costos ambientales ya que las aguas residuales del proceso comúnmente conocidas como aguas mielesson consideradas uno de los mayores contaminantes orgánicos en el sector cafetero, pues muchas de estas aguas retornan a los cauces naturales y son utilizadas nuevamente por las personas localizadas en zonas bajas de dichos cauces (FEDECAFE 2010).

En estudios realizados por Del Panta et al. (2009) se plantea que las aguas residuales de café son una de las principales fuentes que existe un alto riesgo de impacto si no se realiza un tratamiento adecuado con el uso de prácticas de infraestructura para el manejo adecuado de este residuo.

Actualmente la comunidad de la vereda Tres Quebradas está siendo certificada por la ONG RAINFOREST ALLIANCE, que entre sus requisitos tiene evitar el vertimiento directo de las aguas de lavado de café a las fuentes hídricas (Red de Agricultura sostenible 2012). La certificación, que permite el acceso a mercados Internacionales no puede ser otorgada a muchos de los miembros de la comunidad pues no cuentan con un mecanismo eficiente y económico que permita tratar dichas aguas.

La aplicación de la técnica de microorganismos eficientes EM registrada por Higa and Farr (1994), ha sido probada en diferentes tipos de procesos provocando cambios significativos en diversas áreas, como suelos (Lee et al. 2008), (Scheweinsberg-Mickan and Muller 2009), acuicultura (Zhou et al. 2009), producción agrícola (Ncube et al. 2011) y medio ambiente (Qu and Fan 2009), (Yong and Zhong 2010).

Se considera que la aplicación de EM a las 
aguas residuales producto del tratamiento de beneficio húmedo del café trae enormes beneficios, pues estos microorganismos descomponen la materia orgánica de las aguas residuales (Toc et al. 2012).

Pérez (2006), presenta un estudio donde las aguas residuales fueron tratadas con bacterias EM para reducir el nivel de contaminación de estas, demostrando la disminución del nivel de contaminación hasta ocho veces.

En estudios realizados por Delgado (2009), Peñafiel y Donoso (2004) y Terry et al. (2005) se ha observado que el uso de aguas residuales de café tratadas con ME han tenido efectos positivos en la productividad y desarrollo de cultivos, con lo que se podría pensar en utilizar este producto como bioinsumo liquido en muchos cultivos de producción masiva para consumo humano y animal. Otros estudios realizados por el Ministerio de Agricultura de Costa Rica (2010), muestran el uso de ME en obtención de abonos a partir de pulpa de café que son utilizados como abono en los mismos cafetales.

Terry et al. 2005 evaluaron la efectividad agrobiológica de bacterias rizosfericas en el crecimiento, desarrollo y rendimiento en el cultivo del tomate, lo que causó un efecto positivo sobre el crecimiento de las plántulas, así como en el estado nutricional de las plantas, con un rendimiento agrícola superior a un $11 \%$ con respecto a las plantas testigo.

En el marco de la metodología de Aprendizaje Basado en Proyectos de la Institución Universitaria Tecnológica de Comfacauca - UNICOMFACAUCA se realizó este trabajo con el objeto de probar la efectividad de las aguas residualesde café inoculadas con microorganismos eficientes como bioinsumo para la producción de hortalizas, en este caso rábano Raphanus sativus y pepino Cucumis sativus.

\section{MATERIALES Y MÉTODOS}

El proyecto se llevó a cabo en la finca La Aurora vereda Tres Quebradas, ubicada a los $2^{\circ} 53^{\prime} 29,39^{\prime \prime}$ y $76^{\circ} 29^{\prime} 11,12^{\prime \prime}$ jurisdicción del municipio de Santander de Quilichao, Cauca, Colombia. Ubicada en la cordillera central a un altura de 1480 msnm, con una precipitación media de $1800 \mathrm{~mm}$, una temperatura media de $20^{\circ} \mathrm{C}$, una humedad relativa del $65 \%$ y que se clasifica según Holdridge como Bosque Húmedo Premontano (bh - PM).

\section{Técnicasdeobtención delixiviados con MEa partir de pulpa de café.}

Se trabajó con el producto "EM microorganismos eficaces" $₫$ producido por la Fundación de Asesorías para el Sector Rural FUNDASES (Corporación Minuto de Dios). Se trata de una mezcla de bacterias fotosintéticas o fototrópicas Rhodopseudomonas sp, bacterias ácido lácticas Lactobacillus sp y levaduras Saccharomycessp en concentraciones mayores a cien mil unidades formadoras de colonias por $\mathrm{ml}$ de producto y que se encuentran en estado de latencia.

Para hacer el lixiviado se utilizó Bokashi EM que es un material orgánico (salvado de trigo y maíz) que ha sido fermentado por 15 días con EM. El sustrato de replicación utilizado fue pulpa de café a una relación 50:1, es decir $50 \mathrm{~kg}$ de pulpa por $1 \mathrm{~kg}$ de bokashi EM. El recipiente utilizado fue una caneca plástica de doble fondo, con rejilla separadora, tapón 
para extracción de líquidos y tapa de ajuste, (FUNDASES y Acción Social 2009), En primer lugar se fraccionó la pulpa de café para que existiese mayor superficie de contacto, seguidamente se aplicó $50 \mathrm{~g}$ de Bokashi EM al fondo de la caneca sobre la rejilla separadora, posteriormente se aplicó un kg de pulpa de café fraccionada y se siguió colocando una capa sobre otras hasta completar el recipiente; finalmente se presionó para liberar de aire y se tapó herméticamente.

Al cabo de 15 días se obtuvo un lixiviado que se caracterizó por una coloración café claro y un olor suave a vinagre de café, este lixiviado fue aplicado a las aguas resultantes del beneficio de café conocidas como aguas mieles a razón de $50 \mathrm{~cm}^{3} /$ itro $^{-1}$ de agua, que coincide con las recomendaciones técnicas de FUNDASES. Dichas aguas se dejan en inoculación por ocho días para luego utilizarse como bioinsumo.

\section{Evaluación de crecimiento de rábano con diferentes dosis de bioinsumo EM}

Este ensayo se hizo con el fin de determinar la dosis adecuada de bioinsumo EM en variables de crecimiento y productividad del cultivo de rábano - $R$. sativus debido a que es un cultivo de crecimiento rápido y que presenta alta uniformidad genética.

Se utilizó un diseño de bloques completos al azar con cinco tratamientos y tres repeticiones cada uno para un total de 15 unidades experimentales. Los tratamientos evaluados fueron: T1: $5 \mathrm{~cm}^{3} \mathrm{~L}^{-1}$ de bioinsumo; T2: $10 \mathrm{~cm}^{3} \mathrm{~L}^{-}$ 1 de bioinsumo; T3: $30 \mathrm{~cm}^{3} \mathrm{~L}^{-1}$ de bioinsumo; T4: $50 \mathrm{~cm}^{3} \mathrm{~L}^{-1}$ de bioinsumo y TE: testigo (sin bioinsumo). El tamaño de la parcela fue de $1 \mathrm{~m}^{2}$
(1 m X $1 \mathrm{~m}$ ). Una vez preparada la misma se le incorporó un kg del abono orgánico comercial a base de gallinaza a razón de $300 \mathrm{~g} \mathrm{~m}^{2}$.

La densidad de siembra fue de $20 \mathrm{~cm}$ entre calles y $10 \mathrm{~cm}$ entre plantas; para realizar las mediciones se tuvo en cuenta las plantas del interior de cada una de las unidades experimentales con el fin de evitar el efecto de borde, para un total de 38 plantas efectivas. Al final del ensayo (35 días después de siembra) se evaluaron las variables: Numero de hojas: se contó el número total de hojas presentes al momento de cosecha. Longitud total: en la misma se tomó la longitud desde la raíz hasta la hoja más larga. Longitud de raíces: se tomó la medida desde el cuello de la planta hasta la parte engrosada de la raíz napiforme. Diámetro: se tomaron 15 plantas al azar y se mide el diámetro ecuatorial de la muestra. Producción: se midió la producción para la totalidad de las plantas, se estima en $\mathrm{gr}^{2} \mathrm{~m}^{2}$ de peso fresco.

Los resultados se analizaron mediante la utilización de programas estadístico: Statistix en su versión libre; haciendo análisis de varianza y comparación de medias (prueba de Tukey).

\section{Comparación entre bioinsumo y fertilizante de síntesis química en pepino.}

Una vez determinada la dosis ideal en el ensayo anterior realizado con rábano, se procedió a realizar la comparación de la efectividad entre dicha dosis ideal de bioinsumo con un fertilizante de síntesis química de uso común en la región (triple quince) en un cultivo comercial, en este caso pepino ( $C$. sativus) que es sembrado en pequeños lotes y huertas casera y contribuye a mejorar la seguridad alimentaria de los habitantes de la región.

Los tratamientos se organizaron en un 
diseño de bloques completos al azar con tres tratamientos y cuatro repeticiones cada uno, Los tratamientos evaluados fueron: T1: suelo; T2: suelo $+30 \mathrm{~cm}^{3} \mathrm{~L}^{-1}$ de bioinsumo; T3: suelo +10 g triple 15 por planta. El tamaño de cada parcela fue de $24 \mathrm{~m}^{2}$ (6 m X $4 \mathrm{~m}$ ), de las cuales se evaluaron 30 plantas del centro de la parcela para descartar efecto de borde.

Las semillas fueron sembradas según la guía para la siembra de hortalizas de la FAO, la densidad de siembra fue de $1 \mathrm{~m}$ entre calles y $30 \mathrm{~cm}$ entre plantas, se realizaron dos desyerbas manuales y dos controles fitosanitarios. Al momento de cosecha se evaluó longitud de frutos, numero de frutos totales y peso de frutos totales.

Los resultados se analizaron mediante la utilización de programas estadísticos: Statistix en su versión libre; haciendo estadística descriptiva y comparativa entre los tratamientos para las diferentes variables.

\section{RESULTADOS Y DISCUSIÓN}

\section{Evaluación de crecimiento de rábano con diferentes dosis de bioinsumo EM}

La Tabla 1 presenta los resultados obtenidos para las variables evaluadas. análisis de varianza se observó que había diferencias significativas entre los tratamientos, la prueba de comparación de medias de Tukey mostró que existían dos grupos, el primero conformado por el Testigo y los tratamientos T1 y T2, y el otro grupo estaba conformado por los tratamientos T3 y T4. El mayor número de hojas se presenta en los tratamientos que poseen mayor volumen de bioinsumo por litro de agua miel de café, en este caso los tratamientos T3 y $\mathrm{T} 4$ con 30 y $50 \mathrm{~cm}^{3} \mathrm{~L}^{-1}$ respectivamente.

Longitud total de la planta: la prueba de promedios de Tukey muestra que hay diferencias significativas entre tratamientos, la comparación de medias mostró un grupo claramente diferenciado el cual agrupaba a los tratamientos T3 y T4, presentando la mayor longitud.

Longitud de raíces: el análisis de varianza no mostro diferencias significativas para esta característica, siendo el tratamiento T3 el que presentó las raíces más largas.

Diámetro de raíces: el análisis de varianza mostró que no existían diferencias significativas entre los tratamientos utilizados, sin embargo el tratamiento T4 tuvo el mayor diámetro de raíces con $3,5 \mathrm{~cm}$ seguido por el tratamiento $\mathrm{T} 1 \mathrm{con} 3,3 \mathrm{~cm}$.

Número de hojas por planta: al realizar el

Producción: en cuanto a la variable producción,

Tabla 1. Efecto de aplicación de bioinsumo EM a partir de aguas mieles de café en diferentes parámetros de crecimiento y desarrollo de plantas de rábano.

\begin{tabular}{llcccc}
\hline Tratamiento & N de hojas & $\begin{array}{c}\text { Longitud } \\
\text { total }(\mathbf{c m})\end{array}$ & $\begin{array}{c}\text { Longitud de } \\
\text { raíces }(\mathbf{c m})\end{array}$ & $\begin{array}{c}\text { Diámetro } \\
\text { ecuatorial }(\mathbf{c m})\end{array}$ & $\begin{array}{c}\text { Producción } \\
\text { raíz/planta (g) }\end{array}$ \\
\hline TE: $0 \mathrm{~cm}^{3} \mathrm{~L}^{-1}$ & $5,1 \mathrm{a}^{*}$ & $11,68 \mathrm{c}$ & $3,57 \mathrm{a}$ & $3,10 \mathrm{a}$ & $25,1170 \mathrm{a}$ \\
T1: $5 \mathrm{~cm}^{3} \mathrm{~L}^{-1}$ & $5,2 \mathrm{a}$ & $12,10 \mathrm{bc}$ & $3,63 \mathrm{a}$ & $3,30 \mathrm{a}$ & $27,0890 \mathrm{a}$ \\
T2: $10 \mathrm{~cm}^{3} \mathrm{~L}^{-1}$ & $5,1 \mathrm{a}$ & $12,60 \mathrm{~b}$ & $3,93 \mathrm{a}$ & $3,25 \mathrm{a}$ & $28,1278 \mathrm{a}$ \\
T3: $30 \mathrm{~cm}^{3} \mathrm{~L}^{-1}$ & $6,6 \mathrm{~b}$ & $13,48 \mathrm{a}$ & $4,10 \mathrm{a}$ & $2,99 \mathrm{a}$ & $27,2370 \mathrm{a}$ \\
T4: $50 \mathrm{~cm}^{3} \mathrm{~L}^{-1}$ & $6,7 \mathrm{~b}$ & $13,35 \mathrm{a}$ & $3,89 \mathrm{a}$ & $3,50 \mathrm{a}$ & $28,5930 \mathrm{a}$ \\
\hline
\end{tabular}

*Los resultados de tratamientos con letras diferentes en sentido vertical presentaron diferencias significativas (Tukey al $5 \%$ ). 
expresado como peso de la raíz por planta (en gramos) no se encontraron diferencias significativas para esta variable, los pesos frescos de raíces por planta más altos fueron encontrados en el tratamiento T4 con 28,59 cm y el tratamiento $\mathrm{T} 2 \mathrm{con} 28,13 \mathrm{~cm}$. El tratamiento que obtuvo el menor peso fue el testigo.

Los resultados indican que la aplicación del bioinsumo favorece en forma significativa algunos de los parámetros en dosis de 30 ó 50 $\mathrm{cm}^{3} \mathrm{~L}^{-1}$ de agua miel de café. Criollo y García (2009) encontraron que las propiedades microbiológicas de este tipo de bioinsumos e inóculos junto con compost generan mayores desarrollos foliares.

La dosis ideal encontrada concuerda con las recomendaciones técnicas de la "Guía de la Tecnología EM" (Fundases y Acción Social 2009), cita que el pool de EM aumenta la diversidad microbiana del suelo, síntesis de aminoácidos, ácidos nucleícos, vitaminas, hormonas y otras sustancias bioactivas que promueven el crecimiento y desarrollo de las plantas de modo que se incrementan el rendimiento y la calidad de los cultivos.

Dicha guía utiliza como sustrato una mezcla de agua con melaza y no aguas residuales de café. Para este ensayo se determinó que la dosis a aplicar de bioinsumo es de $30 \mathrm{~cm}^{3} \mathrm{~L}^{-1}$, teniendo en cuenta que se tiene similar efecto que cuando se aplican $50 \mathrm{~cm}^{3}$.

\section{Comparación entre bioinsumo y fertilizante de síntesis química.}

Según el análisis estadístico y la comparación de medias, mostrados en la tabla 2, se encontraron diferencias significativas para cada una de las variables evaluadas; como se puede observar el tratamiento T3 de fertilizante es el que mejor respuesta tiene para las características productividad y número total de frutos, seguido por el tratamiento T2 que corresponde a $30 \mathrm{~cm}^{3} \mathrm{~L}^{-1}$ agua de bioinsumo a partir de aguas residuales de café. Por su parte el testigo mostró los valores más bajos, confirmando que el bioinsumo tiene un efecto positivo en los parámetros de productividad evaluados. En cuanto a longitud de frutos se presentaron diferencias estadísticas significativas para los tratamientos. El tratamiento con bioinsumoa partir de aguas mieles de café presentaron los valores más altos.

Los resultados encontrados coinciden con los resultados de Terry et al. (2005) que demostraron la efectividad agrobiológica de microorganismos benéficos a través del estímulo positivo ejercido en el crecimiento, estado nutricional y el rendimiento agrícola del

Tabla 2. Efecto de aplicación de bioinsumo EM a partir de aguas mieles de café en diferentes parámetros de crecimiento y desarrollo de plantas de pepino.

\begin{tabular}{lccc}
\hline \multicolumn{1}{c}{ Tratamiento } & $\begin{array}{c}\text { Longitud de frutos } \\
(\mathbf{c m})\end{array}$ & $\begin{array}{c}\text { Productividad total } \\
(\mathbf{K g})\end{array}$ & Número total de frutos \\
\hline T1: Testigo & $12,8 \mathrm{c}$ & $15,325 \mathrm{c}$ & $117,5 \mathrm{c}$ \\
T2: Bioinsumo & $16,7 \mathrm{~b}$ & $20,475 \mathrm{~b}$ & $139,0 \mathrm{~b}$ \\
T3: Triple 15 & $15,9 \mathrm{a}$ & $22,875 \mathrm{a}$ & $156,5 \mathrm{a}$ \\
\hline
\end{tabular}

*Los resultados de tratamientos con letras diferentes en sentido vertical presentaron diferencias significativas (Tukey al 5\%) 
cultivo de tomate (Lycopersicon esculentum Mil.), por tanto la aplicación de bioinsumo de aguas mieles de café tratadas con ME podría convertirse en una alternativa para el proceso productivo de hortalizas.

\section{CONCLUSIONES}

El bioinsumo a partir de aguas mieles de café inoculadas con microorganismos eficientes se puede convertir en una alternativa potencial para dar un mejor uso de los subproductos del cultivo de café con aplicabilidad en los sistemas de cultivos de hortalizas como biofertilizante.

\section{REFERENCIAS}

Criollo, H. y García, J. 2009. Efecto de la densidad de siembra sobre el crecimiento de plantas de rábano (raphanus sativus $\mathrm{L}$.) bajo invernadero. Revista Colombiana de Ciencias hortícolas 3(2):210-222.

Delgado, Y. 2009. Evaluación de microorganismos eficientes en el cultivo de tomate (Lycopersicon esculentum Mill) en el organopónico de la Universidad de Matanzas. http://monografias.umcc.cu/ monos/2009/AGRONOMIA/m09agr21. pdf [12 Septiembre 2012].

Del Panta, L. Regio, G. y Gil, D. 2009. Estudio sistema de tratamiento de las aguas residuales en Salcedo República Dominicana. www.cafeycaffe.org/web/ index.php [10 Septiembre 2012].

FEDECAFE. 2009. Cartillas cafeteras, tomo 2. El beneficio del café, Colombia, p22.

FEDECAFE. 2010. Sobre el café, un producto especial: Post-cosecha. http://www. cafedecolombia.com/particulares/es/ sobre_el_cafe/el_cafe/ [23 Agosto 2012].

FUNDASES y Acción Social. 2009. Guía de uso de tecnología EM en la agricultura. Corporación Minuto de Dios. Bogotá, p8.

Higa, T. and Farr, J. 1994. Beneficial and effective microorganisms for a sustainable agriculture and environment. International Nature Farming Research Center. Atami, Japan, p16.

Lee, C. Muhamad, I., Razali, F. and Khamis, A. 2008. Application of beneficial microorganisms on agriculture. In: Special Topics In Bioprocess Engineering 3:139-162.

Ministerio de Agricultura de Costa Rica. 2010. Manual de buenas prácticas de manufactura en el beneficio UNDECAF. www.mag.go.cr/bibliotecavirtual/ a00213.pdf [29 Agosto 2012].

Ncube, L. Nyari, P. y Brutsc, M. 2011. Agronomic suitability of effective microorganisms for tomato production. http:// www.academicjournals.org/AJAR Febrero 2013].

Peñafiel, B. y Donoso, M. 2004. Evaluación de Diferentes Dosis de Microorganismos Eficientes (Me) en el Cultivo de Pepino (Cucumis Sativus) Híbrido Atar Ha435. Tesis ingeniería Agropecuaria. Universidad de Guayaquil, Ecuador, p129.

Pérez, M. 2006. La finca Irlanda: modelo 
organizacional de la agricultura orgánica-biodinámica, sustentable y responsable. Cambio y estrategia en una organización cafetalera en el soconusco. Tesis de Doctorado. Universidad Autónoma Metropolitana. México.

Qu, J. and Fan, M. 2010. The Current State of Water Quality and Technology Development for Water Pollution Control in China. http://www.tandfonline.com/ doi/pdf/10.1080/10643380802451953 [21 Febrero 2013].

Red de Agricultura Sostenible - RAS. 2012. Indicadores locales para la producción de café sostenible en Colombia. www. sanstandards.org [30 Agosto 2012].

Scheweinsberg-Mickan,M.yMuller,T.2009. Impact of effective microorganisms and other biofertilizer on soil microbial chararacteristics, organic - matter decomposition and plant growth. http:// onlinelibrary.wiley.com/doi/10.1002/ jpln.200800021/abstract [22 Febrero 2013].

Terry, E., Leyva, A. y Hernández, A. 2005.
Microorganismos benéficos como biofertilizantes eficientes para el cultivo del tomate (Lycopersicon esculentum Mill) Revista Colombiana de Biotecnología 7(2):4754.

Toc,R.,Castillo,R.yTrabanino,R.2012.Efecto de los Microorganismos Eficientes (ME) en las Aguas Residuales de la Granja Porcina de Zamorano, Honduras. Tesis Ingeniero Agrónomo. http://bdigital. zamorano.edu/handle/11036/1039 [26 Febrero 2013].

Yong, Y. and Zhong, J. 2010. Recent advances in biodegradation in China: New microorganisms and pathways, biodegradation engineering, and bioenergy from pollutant biodegradation. http://www.sciencedirect.com/science/ article/pii/S1359511310001492 Marzo 2013].

Zhou, Q., Li, K. and Liu, X. 2009. Role and functions of beneficial microorganisms. http://www.ncbi.nIm.nih.gov/ pubmed/19261470 [28 Febrero 2013]. 